Research Article

\title{
Analysis of the Risk Path of the Pipeline Corridor Based on System Dynamics
}

\author{
Bao-Jiang Han, ${ }^{1,2}$ Yu-Sheng Jiang, ${ }^{1}$ Zihao Wang, ${ }^{3}$ Daqing Gong $\mathbb{D},^{3}$ \\ Hua Jiang $\mathbb{D}^{1}{ }^{1}$ and Ping-lin Jiang $\mathbb{D}^{1}$ \\ ${ }^{1}$ School of Mechanics and Civil Engineering, China University of Mining \& Technology, Beijing 100083, China \\ ${ }^{2}$ Beijing Jingtou Urban Utility Tunnel Investment Co., Ltd, Beijing 100027, China \\ ${ }^{3}$ School of Economics and Management, Beijing Jiaotong University, Beijing 100044, China
}

Correspondence should be addressed to Hua Jiang; jianghua@cumtb.edu.cn

Received 17 January 2021; Revised 12 March 2021; Accepted 22 May 2021; Published 6 July 2021

Academic Editor: Zhixiong Li

Copyright $(92021$ Bao-Jiang Han et al. This is an open access article distributed under the Creative Commons Attribution License, which permits unrestricted use, distribution, and reproduction in any medium, provided the original work is properly cited.

Under the background of China's continuous promotion of urbanization, urban underground integrated pipeline corridor has become an inevitable trend of future urban integrated management. After the completion of the pipeline corridor, how to effectively manage its risks in operation and maintenance management has become a topic at this stage. In this paper, through the combination of the classical AHP method and DSM method, based on a large number of literature studies, the risk relationship system of the integrated pipe corridor is constructed. AnyLogic software is applied to simulate the system dynamics, analyze the impact of dynamic changes of each risk factor on the risk accident of the integrated pipe corridor, carry out uncertainty reasoning from multiple perspectives, and realize the evaluation and analysis of the accident risk of the integrated pipe corridor. The results of the study could provide targeted support tools for integrated pipeline corridor risk operation and maintenance management.

\section{Introduction}

Since the reform and opening up, China's economy has shown a trend of rapid growth. Under this trend, China's urbanization rate has also been continuously increasing from $17.92 \%$ in 1978 to the current $58.52 \%$. The general increasing urbanization rate will become an inevitable trend to exceed $60 \%$ or even $70 \%$ in the future. Therefore, the rapid increase in the urbanization rate has brought about considerable challenges to the construction of supporting infrastructure in cities and towns. For example, water, electricity, communications, sewage treatment, and other resources transported through pipelines necessary for the daily life of urban residents will increase exponentially, and the line network formed by the intertwining of various pipelines will become incomparable. The complexity of the traditional route pattern is no longer applicable, so the form of urban underground pipe gallery has come into being. Driven by both national policy encouragement and urban management upgrades, China's urban underground comprehensive pipeline corridors have been constructed or put into operation in 167 cities in 31 provinces, autonomous regions, and municipalities directly under the Central Government. As of June 2017, the total mileage of integrated pipeline corridors in my country has reached about 5,000 kilometers, and the total mileage of integrated pipeline corridors that have been put into use is about 1,000 kilometers. It can be seen that urban underground comprehensive pipeline corridors have been widely used in my country. However, although the pipe corridors of thousands of kilometers have been built, how to effectively manage and carry out operation and maintenance is still a problem.

After a large number of pipe corridors are put into operation, the safety operation and maintenance risks and related countermeasures of the comprehensive pipe corridor have also attracted more and more attention from the government and scholars. Julian et al. [1] coordinated management in the comprehensive pipe corridor operation for the feasibility and necessity of period management, a 
method based on the combination of expert system and color coding is proposed, and the potential key risk factors of the integrated pipeline gallery are analyzed by the analytic hierarchy process. Chen et al. [2] analyzed the potential disaster risk factors in the operation and maintenance of the integrated pipeline gallery and then constructed a disaster risk assessment model based on Bayesian networks. Li et al. [3] used the DEMATEL method to sort the degree of influence and importance of the identified risk factors and then made recommendations for risk management of the integrated pipeline gallery. Jang and Kim [4] studied the gas explosion caused by gas leakage and unknown ignition in the integrated pipeline gallery. Wang [5] analyzed the potential influencing factors of underground comprehensive pipe gallery fire based on the three elements of combustion and gave the setting range of fire warning for the operation and maintenance of comprehensive pipe gallery. Francesca and Matjaž [1] proposed a simplified risk-targeted decisionmaking model to verify that the seismic performance of infrastructure components reaches the operating limit state.

However, the risk and safety issues that occurred during the operation and maintenance of the pipeline gallery are relatively complicated due to the collection of pipelines and the closed environment, and the corresponding experience is often lacking, resulting in improper handling. This article combines the classic Analytic Hierarchy Process (AHP) method with the Design Structure Matrix (DSM) method and, through a large amount of literature research, constructs a set of risk relationships system for a comprehensive pipeline gallery, using AnyLogic Software to carry out system dynamics simulation, analyze the impact of the dynamic changes of various risk factors on the risk accidents of the comprehensive pipeline gallery, carry out multiangle uncertainty reasoning, and realize the assessment and analysis of the comprehensive pipeline gallery accident risk. The results could be used in the risk operation and maintenance management of the pipe gallery.

\section{Related Work}

The safety operation and maintenance risks and related countermeasures of the comprehensive pipe corridor, etc., had become a necessary research work. The article will provide an overview of the following aspects.

Risk factors: Ghosh et al. evaluated a large-scale underground metro project in Thailand based on these risk variables, using principal component analysis to assess the responses obtained within the project organization and to understand the potential structure of key risk factors for the metro project [6]. Eybpoosh et al. used structural equation modeling (SEM) techniques to identify 36 interrelated risk paths and to assess the overall impact of each vulnerability factor and risk path on cost overruns [7]. Chien et al. used decision testing and evaluation laboratory methods to employ 13 risk factors related to technical, managerial, personnel, financial, and legal aspects of BIM, based on questionnaires distributed to architects, engineering consultants, academics, and construction companies in the architectural, engineering, and construction industries in
Taiwan, and ultimately the relationship between the risk factors was determined [8].

Research methods: Yang et al. introduced the DEMATEL method to build a relational graph structure to elucidate the interrelationships between several criteria and visualize the complex causal relationships between criteria in a real system and further applied the causal graph of the DEMATL method based on the basic concepts of the article to help substitute performance to the desired level required by each criterion [9]. Tsai et al.'s model first applies the Decision Experimentation and Assessment Laboratory (DEMATEL) method to construct the interrelationships between the required criteria for the organization, obtains the criteria weights through ANP, and finally obtains the best alternative with the required organizational benefits by making the best use of the limited resources [10]. In the article, Wu proposes an effective combined ANP and DEMATEL method-based solution to help companies that need to evaluate and select a knowledge management strategy [11].

Management designs: as there are many participating entities in each integrated pipe corridor project, the management and ownership and other relationships are very complex, which brings about many problems in terms of safety management of the integrated pipe corridor during operation [12]; and because most of the integrated pipe corridor projects contain utility facilities with different functions, such as sewage, gas, and electricity, the different responsibilities attributed to them and the highly complex coupling of the facilities bring about great difficulties to the operation and maintenance management [13]. Julian et al. [14] proposed a method based on a combination of expert systems and color coding by analyzing the feasibility and necessity of collaborative management in the operation phase of the integrated corridor and analyzed the potential key risk factors of the integrated corridor using hierarchical analysis. Canto-Perello et al. [15] proposed an expert system combining color-coding, Delphi, and hierarchical analysis to analyze the criticality and threat of integrated corridors to support the planning of safety policies for urban underground facilities. Canto-Perello and Curiel-Esparza pointed out the innovative nature and advantages in the organization of integrated pipe corridors, thus further suggesting that safety management should be an important basis for decisions related to integrated pipe corridors at a later stage [12]. Jang and Jung presented a gas leak in a gas pipeline inside a utility tunnel, thus leading to an unknown explosion causing a methane explosion, and calculated the extent of the impact of the explosion on securing the corridor and its internal facilities, further suggesting the need to design additional safety measures [4].

Risk evaluation: in the research on risk evaluation of integrated pipeline corridors, Canto-Perello et al. proposed an expert system combining color-coding, Delphi, and hierarchical analysis methods to analyze the criticality and threat of integrated pipeline corridors, which was used to support the planning of safety policies for urban underground facilities [14]; Jang et al. studied the risk of gas leakage and unknown ignition in integrated pipeline corridors due to gas explosions [4]; Wang et al. constructed a 
risk evaluation model for integrated pipe corridor PPP projects, identified risk factors for utility based on a questionnaire survey, and then designed a risk evaluation index system and used an optimized fuzzy integrated rating method for risk evaluation [15]; He et al. proposed a new fire risk assessment method within integrated pipe corridors in the absence of historical cable fire data, fuzzy theory was used to calculate the failure probability of the main events of cable fires, fuzzy inference was performed using a weighted fuzzy Petri net, and a numerical simulation method was used to quantify the losses caused by cable fires so as to quantify the risk of cable fires [16]; Ding et al. applied a fault tree model to influence the urban underground integrated pipeline corridor project PPP model risk and concluded that the project risk has a greater impact on the factors and found that the application of the PPP model in underground integrated pipeline corridors is more suitable for developed regions [17]; Zhou et al. and Seong et al., on the other hand, analyzed the key risks of urban integrated pipeline corridors and their ratings from the actual situation of Chinese as well as Korean cities, respectively. As early as 2011, Sousa et al. proposed a method to systematically access and manage tunnel-related risks by combining a geological prediction model with a construction strategy decision model to predict the geology prior to tunnel construction to select the least risky construction strategy among different construction strategies [18]; Golam et al. proposed a Bayesian belief network for assessing the risk of failure of metal water pipes model that can rank water supply trunk pipes in distribution networks to identify vulnerable and sensitive pipes for rational water supply management [19]; Zhang et al. proposed a method for tunnel fire safety risk analysis based on fuzzy Bayesian networks [20]; Khwaja et al. proposed a new public-private partnership based on fuzzy integral infrastructure project (PPP project) risk assessment method to help stakeholders make risk management decisions [21]; Wu et al. developed a cloud model-based risk assessment model for shield construction in underground tunnels, which effectively addressed the stochastic uncertainty and fuzzy uncertainty of indicator factors [22]; in addition to these, the literature [23-25] also implemented dynamic analysis for underground tunnels and subsea tunnels; and the dynamic analysis of cable risks was realized. Francesca et al. [1] proposed a simplified risk-based decision model for verifying the seismic performance of infrastructure components up to the operational limit state. Fan et al. [26] analyzed the potential disaster risk factors during the operation and maintenance of integrated corridors and then constructed a Bayesian network-based disaster risk assessment model.

However, the risk and safety issues that occurred during the operation and maintenance of the pipeline gallery are relatively complicated due to the collection of pipelines and the closed environment, and the corresponding experience is often lacking, resulting in improper handling. As the integrated pipeline gallery is put into use, the risk status also changes and develops dynamically with the time axis. Different geological conditions, pipeline settings, management issues, and other objective factors will cause different risk sources; most of the current risk assessment methods are calculated based on static data. The following factors should be considered: the dynamic combination of risk assessment over time, the complexity of the risk factors of the integrated pipeline gallery, the dynamic characteristics of each risk factor, and the lack of reasoning about the impact of its changes over time on the accident.

\section{Research Methods}

The AHP method is to divide the various factors in complex issues into interconnected and orderly levels to make them organized. According to the subjective judgment structure of a certain objective reality (mainly a pairwise comparison), the expert opinions and the analyst's objective judgment are combined. The results are directly and effectively combined to quantitatively describe the importance of comparing the two elements of a level. DSM is a project management tool that helps business analysis and project management. DSM makes the management process easier to visualize, allows to identify and represent elements in the project, tracks periodic task dependencies and task flow, and helps analyze how and where to improve the dependencies between systems. This article combines the AHP method with DSM method to construct a set of risk relationships system for a comprehensive pipeline gallery.

3.1. AHP. The Analytic Hierarchy Process, abbreviated as AHP, refers to a decision-making method that decomposes the elements that are always related to decision-making into goals, criteria, plans, and other levels and then performs qualitative and quantitative analysis on this basis. This method is based on the application of network system theory in the United States Department of Defense research on the topic of "power distribution based on the contribution of various industrial sectors to national welfare" in the early 1970s. This multiobjective comprehensive evaluation method is a level weight decision analysis method.

The analytic hierarchy process considers the complex multiobjective decision-making problem as a system, the goal is decomposed into multiple goals or criteria and then decomposed into multiple levels of multiple indicators (or criteria, constraints), and the level is calculated through the fuzzy quantitative method of qualitative indicators. Single ranking (weight) and total ranking are used as a systematic method for target (multi-index) and multiplan optimization decision-making.

The analytic hierarchy process is to decompose the decision-making problem into different hierarchical structures according to the order of the general goal, the subgoals of each level, the evaluation criteria, and the specific investment plan and then to use the method of solving the eigenvectors of the judgment matrix to obtain the various factors. The priority of an element to an element at the previous level and finally the weighted sum method is to recursively merge the final weight of each alternative plan to the overall goal, and the one with the largest final weight is the optimal plan. The analytic hierarchy process decomposes the problem into different components according to the 
nature of the problem and the overall goal to be achieved and combines the factors at different levels according to the interrelationship between the factors and the affiliation relationship, forming a multilevel analysis structure model, so as to finally make the problem boiled down to the determination of the relatively important weight of the lowest level (plans, measures, etc., for decision-making) relative to the highest level (the overall goal) or the arrangement of the relative order of superiority and inferiority.

3.1.1. Build a Hierarchy Model. Divide decision-making goals, consideration factors (decision criteria), and decision objects into the highest, middle, and lowest levels according to their mutual relationship and draw a hierarchical structure diagram. The highest level refers to the purpose of decision-making and the problem to be solved. The lowest level refers to alternatives when making decisions. The middle layer refers to the factors to be considered and the criteria for decision-making. For the two adjacent layers, the upper layer is called the target layer and the lower layer is the factor layer.

3.1.2. Construct a Judgment (Pair Comparison) Matrix. When determining the weights between various factors at various levels, if it is only a qualitative result, it is often not easy to be accepted by others. Therefore, Saaty et al. proposed the consistent matrix method, which does not compare all factors together but compares the pair factors. For comparison, a relative scale is used at this time to minimize the difficulty of comparing various factors with different properties to improve accuracy. For example, for a certain criterion, compare the schemes under it in pairs, and rank according to its importance. $a_{i j}$ is the comparison result of the importance of element $i$ and element $j$. Table 1 lists the nine importance levels and their assignments given by Saaty. The matrix formed by the pairwise comparison results is called the judgment matrix. The judgment matrix has the following properties in equation

$$
a_{i j}=\frac{1}{a_{i j}} .
$$

The scaling method of the judgment matrix element $a_{i j}$ is shown in Table 1.

3.1.3. Hierarchical Order and Its Consistency Check. The eigenvector corresponding to the largest eigenvalue $\lambda$ max of the judgment matrix is normalized (to make the sum of the elements in the vector equal to 1 ) and then denoted as $W$. The elements of $W$ are the ranking weights of the relative importance of the factors at the same level to the factors of the previous level. This process is called single-level ranking. Whether the order of the level list can be confirmed requires a consistency test. The so-called consistency test refers to determining the allowable range of inconsistency for $A$. Among them, the only nonzero characteristic root of the $n$ th-order consistent matrix is $n$; the largest characteristic root
TABLE 1: The scaling method of the judgment matrix element $a_{i j}$.

\begin{tabular}{lc}
\hline Rating & Level \\
\hline 1 & Equally important \\
3 & Slightly important \\
5 & Important \\
7 & Very important \\
9 & Extremely important \\
$2,4,6$, and 8 correspond to the intermediate level of each level \\
\hline
\end{tabular}

of the $n$-th-order reciprocal matrix $A$ is $\lambda \geq n$; if and only if $\lambda=n, A$ is a consistent matrix.

Since $\lambda$ continuously depends on $a_{i j}$, the more $\lambda$ is larger than $n$, the more serious the inconsistency of $A$ is, and the consistency index is calculated by CI. The smaller the CI, the greater the consistency. The eigenvector corresponding to the largest eigenvalue is used as the weight vector of the degree of influence of the compared factor on a certain upper-level factor. The greater the degree of inconsistency is, the greater the judgment error is caused. Therefore, the value of $\lambda-n$ can be used to measure the inconsistency of $A$. Define the consistency index in the following equation:

$$
\mathrm{CI}=\frac{\lambda-n}{n-1} .
$$

In equation (2), when $\mathrm{CI}=0$, there is complete consistency; when CI is close to 0, there is satisfactory consistency; the larger the CI, the more serious the inconsistency. In order to measure the size of CI, the random consistency index RI is introduced in the following equation:

$$
\mathrm{RI}=\frac{\mathrm{CI}_{1}+\mathrm{CI}_{2}+\mathrm{CI}_{3}+\cdots \mathrm{CI} n}{n}
$$

In equation (3), the random consistency index RI is related to the order of the judgment matrix. In general, the larger the order of the matrix, the greater the probability of random deviation from consistency. Considering that the deviation of consistency may be caused by random reasons, when testing whether the judgment matrix has satisfactory consistency, we should compare CI and random consistency index RI to obtain the test coefficient CR. The formula is as follows:

$$
\mathrm{CR}=\frac{\mathrm{CI}}{\mathrm{RI}}
$$

In equation (4), if $\mathrm{CR}<0.1$, the judgment matrix is considered to pass the consistency test; otherwise, there is no satisfactory consistency.

3.2. Risk Structure Matrix. Identification is the first step in determining the causal relationship between risks. The DSM method proposed by Steward has been proven to be a practical tool for expressing and analyzing the relationships and dependencies between system components. In our research, we combined the concept of DSM with risk in the context of integrated pipeline corridor operation and maintenance management. 
There may be multiple links of different nature between the two risks. They are expressed as potential causal relationships between risks. Risk interaction is considered to be a possible precedent relationship between two risks. We have defined the risk structure matrix (RSM). When there is a link from $R_{j}$ to $R_{i}$, it is a binary sum square matrix with $R S M_{i j}=1$ or 0 . This does not solve the problem about the possibility of this interaction or the impact assessment. Its role is only to determine the existence of the relationship between $R_{j}$ and $R_{i}$. It is a qualitative rather than quantitative judgment.

$p$ represents a quantitative probability measure, and a qualitative scale is usually used to express the probability of 5 to 10 levels. Risk Interaction Assessment compared with the binary structure of the risk network used to assist decisionmaking, and the digital structure matrix can provide more detailed information. Evaluation is the process of measuring and evaluating the strength of the link between risks. Two methods can be used for estimation: direct evaluation and relative evaluation. One or more experts directly evaluate each potential interaction based on their experience and/or expertise. Relative assessment consists in comparing the causes (or effects) of a single risk with multiple interactions. This involves using the principle of pairwise comparison in the analytic hierarchy process (AHP) developed by Saaty. Marle has developed an AHP-based assessment method, and the main principles for obtaining the numerical value of the risk interaction strength are described in the following paragraphs.

Step 1: first, generate a risk judgment sparse matrix RSN based on the actual situation in reality, mainly to determine whether the potential relationship between various risk factors exists. Then decompose the individual subproblems. For each risk $R_{i}$, separate the risks related to the $R_{i}$ column (possible effects) and the $R_{i}$ row (possible causes). This recognition enables to generate binary cause (or effect) vectors about risk $R_{i}$, called $B C V \mid R_{i}$ and $B e v \mid R_{i}$, respectively, indicating that a certain risk factor $R_{i}$ is affected by other risk factors.

Step 2: to assess the relative strength of a risk $R_{i}$ (CCM) $R_{i}$ and $\mathrm{ECM} \mid R_{i}$, respectively), two matrices (cause or effect comparison matrix) need to be established. Then apply the AHP method to comprehensively score the risk matrix. AHP is based on the use of pairwise comparisons, which led to the formulation of the ratio table. In our example, we have two parallel pairwise comparison processes to run. The first is the row ranking of each project risk. The criterion for evaluating alternatives is the contribution to the risk input of $R_{i}$. In other words, for each pair of risks $R_{j}$ and $R_{k}$ compared (hence, $R S M_{i j}=R S M_{i k}=1$ ), the user should evaluate which risk is more important for $R_{i}$ based on the probability of triggering $R_{i}$. Due to the use of the traditional AHP scale, these assessments are expressed in numerical values. The second is the ranking by column based on the same principle.

Step 3: determine the weight vector of each matrix. Now calculate the eigenvectors of each matrix $E C M \mid R_{i}$ and $C C M \mid R_{i}$. It enables to find the main feature vectors corresponding to the largest feature value. They are called numerical causal vectors and are relative to a type of risk $R_{i}\left(N C V_{i}\right.$ and $\left.N E V_{i}\right)$. Due to the AHP consistency index, the consistency of the results should be tested.

Step 4: summarize eigenvectors for each risk $R_{i}$, and summarize the numerical causality vectors (NCV and $\mathrm{NEV}$ ) into the numerical causality matrix (NCM and NEM). The $i$-th row of NEM corresponds to the eigenvector of $C C M \mid R_{i}$, which is associated with its maximum eigenvalue. The $j$ th column of NCM corresponds to the eigenvector of $E C M \mid R_{j}$, which is associated with its maximum eigenvalue.

Step 5: summarize the results. The first two matrices are summarized into a risk number matrix (RNM), whose values evaluate the relative strength of local interactions. RNM is defined by the geometric weighting operation in equation (1) (based on the assumption that both estimates can be considered equivalent in terms of causality). We chose the geometric mean rather than the arithmetic mean because it tends to support the equilibrium value (between two evaluations).

$\mathrm{RNM}_{i j}$ defines the strength of causal interaction. RNM combines interaction based on cause and effect, so it can integrate the existence and strength of local priority relationships between risks. This helps to avoid any bias or misassessment that may occur when viewing the problem from a single perspective. In the risk network model, the value of causal interaction in RNM can also be interpreted as the transition probability between risks. For example, if the element $\operatorname{RNM}(4,3)$ is equal to 0.25 , under the condition that risk 3 is activated, the probability that risk 4 is considered to be derived from risk 3 is $25 \%$.

\section{Analysis of Safety Risk Factors of Integrated Pipeline Gallery Operation and Maintenance Management}

4.1. Factor Identification. Before studying the risk management of an integrated pipe gallery, we must first clarify the types of risk factors that affect the overall safety of the pipe gallery. Risk factors refer to any event that increases the frequency or severity of risk accidents. The more conditions that constitute a risk factor are, the greater the possibility of loss will be and the more serious the loss will be. The traditional methods of identifying risk factors mainly include brainstorming method, SWOT analysis method, Delphi method, scenario analysis method, risk table checking method, and literature research method.

According to the relevant laws and regulations on the operation and maintenance of the integrated pipe gallery, the corresponding operation and maintenance management requirements documents, and the predecessor's literature on the risk research of the operation and 
maintenance of the integrated pipe gallery, a comprehensive research on the risk factors table of the integrated pipe gallery is obtained, and the classified risk factors are obtained. However, the results of the risk factors obtained are exchanged with the operation and maintenance experts of the integrated pipeline gallery, and, finally after modification, summarization, and sorting operations, a total of 5 types of primary risks are obtained, which are recorded as $R_{i}, 20$ types of secondary risks as $r_{i}$, and the relevant basis for the conclusion.

Since there are relatively few researches on the operation and maintenance risks of pipeline corridors and there are relatively few reference samples for summarizing the risk factors of pipeline corridors, this article considers two types of literature to study, respectively: the literature on comprehensive pipeline corridor risks and a variety of large-scale project operation and maintenance management risk documents to extract the first-level risk factors and second-level risk factors that occur frequently. This article analyzes a total of 8 documents of various types in the aforementioned fields and lists the risk factors covered by them with higher frequency, their frequency, and the cited documents (shown in Table 2).

4.2. Path Determination of the Risk System. After determining the various risk factors in the operation and maintenance process of the integrated pipeline gallery, we must deal with the various risk factors according to the improved AHP mentioned earlier and obtain the interactive relationship among all factors, paving the way for the next analysis with simulation.

To determine the potential connections between 5 firstlevel risks and 20 second-level risks and the corresponding path weights, we should analyze from the following three aspects: the relationship between each first-level risk and the comprehensive risk of the pipe gallery, the relationship between primary risk and its corresponding secondary risk, and the potential relationship between secondary risks. This part is divided into three steps in total.

Here, the calculation of the risk relationship matrix between each primary risk and the comprehensive risk of the pipe gallery is taken as an example. The judgment matrix is based on interviews with relevant experts and comprehensively scored by experts. The scoring level is from 1 to 9 , where $1,3,5,7$, and 9 correspond to five different levels of importance, and 2, 4, 5, 6, and 8 are the intermediate values between the five levels.

The experts are interviewed in the form of online questionnaires, and the data are processed by the experts on the answer results of the questionnaires, so that the judgment matrix of the risks of each primary risk and the comprehensive risk of the pipe gallery is obtained as follows. Then the judgment matrix is calculated. The calculation principle is as described above. Here, the $\mathrm{R}$ language is used to program the entire analytic hierarchy process. You only need to input the target matrix to obtain the weight vector and the result of the consistency check.

$$
\left(\begin{array}{cccccc} 
& R_{1} & R_{2} & R_{3} & R_{4} & R_{5} \\
R_{1} & 1 & 3 & 3 & 5 & 2 \\
R_{2} & 0.3 & 1 & 3 & 4 & 1 \\
R_{3} & 0.3 & 0.3 & 1 & 0.25 & 0.3 \\
R_{4} & 0.2 & 0.25 & 4 & 1 & 1 \\
R_{5} & 0.5 & 1 & 3 & 1 & 1
\end{array}\right) .
$$

Finally, according to the calculation of the program, the corresponding weights of $R 1$ to $R 5$ are $0.415,0.218,0.062$, 0.122 , and 1.183 , respectively. The consistency index $C I$ is 0.099 , which meets the confidence level (less than 0.1), and the random consistency index $C R$ is 0.089 . The confidence level is met, so it can be determined that the result has passed the consistency test, and the obtained weight vector is considered usable. Thus, the first-level risk framework structure diagram corresponding to the comprehensive risk of the pipe gallery can be drawn.

Using the same method as above, the weight vector between each secondary risk and the corresponding primary risk can be obtained. The judgment matrix, weight vector, and consistency test results are shown in Table 3.

According to the obtained results, except for the judgment matrices of $R_{2}$ and $R_{5}$, the other judgment matrices have passed the consistency test. After analysis, it is believed that $R 2$ environmental risk factors are affected by experts' subjective factors. The error is relatively large: $R_{5}$ is the risk of the pipe gallery. The interviewed experts are mainly relevant personnel engaged in the operation and maintenance management of the integrated pipe gallery. Experts say that the risks related to the main body of the pipe gallery are more related to the civil construction of the pipe gallery, so this is not what they excel. Therefore, for $R_{2}$, by asking different experts and comprehensively scoring their opinions, the judgment matrix is reobtained; for $R_{5}$, the judgment matrix is reobtained by asking relevant experts engaged in the civil construction of the integrated pipe corridor. The two judgment matrices obtained again are shown in Table 4.

The result of the consistency test shows that the improved judgment matrix successfully passed the consistency test, so the optimization work is considered successful. After determining the weights between different levels of risks, the next step is to confirm the relevant paths between the secondary risks and their path coefficients. The method of confirming the path and confirming the path coefficient is the same as the method described above in this article. It is through interviews with experts to determine the 0,1 construction matrix of the risk path. According to the calculation method introduced previously, 20 secondary risks are obtained. The path coefficients, the final related paths, and path coefficients are shown in Table 5.

\section{Experimental Design and Results}

The survey was mainly conducted for the design, construction, operation, and maintenance of pipeline corridors and university researchers. 120 questionnaires were distributed to the relevant personnel for the integrated pipeline 
TABLE 2: Comprehensive pipeline gallery risk system.

\begin{tabular}{|c|c|}
\hline Primary risk & Secondary risk \\
\hline Management team risk $R_{1}$ & $\begin{array}{c}\text { Team information level } r_{1} \\
\text { Team personnel level } r_{2} \\
\text { Personnel safety awareness level } r_{3} \\
\text { Information management level } r_{4} \\
\text { Personnel mental state } r_{5}\end{array}$ \\
\hline Environmental factor risk $R_{2}$ & $\begin{array}{c}\text { Geological disaster impact } r_{6} \\
\text { Illegal invasion of people and animals } r_{7} \\
\text { Third-party construction damage } r_{8} \\
\text { Temperature, humidity, and chemical gas concentration in the corridor } r_{9}\end{array}$ \\
\hline Ingress pipeline risk $R_{3}$ & $\begin{array}{c}\text { Pipeline leak } r_{10} \\
\text { Valves, pipe fittings, etc., are not maintained in time } r_{11} \\
\text { Cracks, pipeline deviation, pipeline slippage, etc., caused by uneven settlement } r_{12} \\
\text { Explosion and fire caused by pipeline } r_{13}\end{array}$ \\
\hline Auxiliary facility risk $R_{4}$ & $\begin{array}{l}\text { Lighting, ventilation, and drainage facilities } r_{14} \\
\text { Above-ground and underground information is not linked } r_{15} \\
\text { Risk sensing facilities in the corridor } r_{16}\end{array}$ \\
\hline Risk of pipe gallery $R_{5}$ & $\begin{array}{c}\text { Design and construction do not meet operation and maintenance requirements } r_{17} \\
\text { Uneven settlement caused by construction problems cited } r_{18} \\
\text { Unreasonable overall construction design } r_{19} \\
\text { Information transfer between management center and gallery } r_{20}\end{array}$ \\
\hline
\end{tabular}

TABLE 3: Consistency test of the risk path of integrated pipeline gallery.

\begin{tabular}{|c|c|c|c|c|}
\hline Level 1 risk category & Level 1 risk category & Level 1 risk category & Level 1 risk category & Level 1 risk category \\
\hline$R_{1}$ & $\left.\begin{array}{cccccc} & r_{1} & r_{2} & r_{3} & r_{4} & r_{5} \\
r_{1} & 1 & 1 & 3 & 2 & 5 \\
r_{2} & 1 & 1 & 2 & 0.3 & 4 \\
r_{3} & 0.3 & 0.5 & 1 & 0.5 & 2 \\
r_{4} & 0.5 & 3 & 2 & 1 & 3 \\
r_{5} & 0.2 & 0.25 & 0.5 & 0.3 & 1\end{array}\right)$ & $\left(\begin{array}{lllll}0.342 & 0.206 & 0.118 & 0.269 & 0.065\end{array}\right)$ & $0.057-0.051$ & Yes \\
\hline$R_{2}$ & $\left(\begin{array}{ccccc} & r_{6} & r_{7} & r_{8} & r_{9} \\
r_{6} & 1 & 5 & 6 & 3 \\
r_{7} & 0.2 & 1 & 0.2 & 0.3 \\
r_{8} & 0.17 & 5 & 1 & 4 \\
r_{9} & 0.3 & 3 & 0.25 & 1\end{array}\right)$ & $\left(\begin{array}{llll}0.564 & 0.061 & 0.249 & 0.126\end{array}\right)$ & $0.172-0.192$ & No \\
\hline$R_{3}$ & $\left(\begin{array}{ccccc} & r_{10} & r_{11} & r_{12} & r_{13} \\
r_{10} & 1 & 3 & 4 & 0.3 \\
r_{11} & 0.3 & 1 & 0.3 & 0.2 \\
r_{12} & 0.25 & 3 & 1 & 0.3 \\
r_{13} & 2 & 5 & 3 & 1\end{array}\right)$ & $\left(\begin{array}{llll}0.316 & 0.074 & 0.139 & 0.472\end{array}\right)$ & $0.043-0.048$ & Yes \\
\hline$R_{4}$ & $\left(\begin{array}{cccc} & r_{14} & r_{15} & r_{16} \\
r_{14} & 1 & 0.2 & 0.3 \\
r_{15} & 5 & 1 & 2 \\
r_{16} & 3 & 0.5 & 1\end{array}\right)$ & $\left(\begin{array}{lll}0.106 & 0.584 & 0.310\end{array}\right)$ & $-0.014-0.024$ & Yes \\
\hline$R_{5}$ & $\left.\begin{array}{ccccc} & r_{17} & r_{18} & r_{19} & r_{20} \\
r_{17} & 1 & 0.14 & 6 & 5 \\
r_{18} & 7 & 1 & 0.3 & 5 \\
r_{19} & 0.17 & 3 & 1 & 4 \\
r_{20} & 0.2 & 0.2 & 0.25 & 1\end{array}\right)$ & $\left(\begin{array}{llll}0.302 & 0.380 & 0.252 & 0.067\end{array}\right)$ & $0.809-0.898$ & No \\
\hline
\end{tabular}

corridors in Beijing, of which 105 were valid, with an effective rate of $87.5 \%$. The expert survey method was applied to collect experts' opinions on the levels of the above risk factors and the weights to which the risk factors belong. Due to the dynamics and uncertainty of risk factors related to pipelines, the sample data often have some hidden variables that cannot be observed, so this paper adopts the iterative convergence algorithm with missing values for the sample, EM algorithm for parameter learning, and through multiple iterations to make the model parameters converge to the maximum likelihood estimation and finally get the conditional probability distribution, so as to determine the probability of the distribution of each disaster risk factor level. 
TABLE 4: Risk judgment matrix of comprehensive pipe gallery.

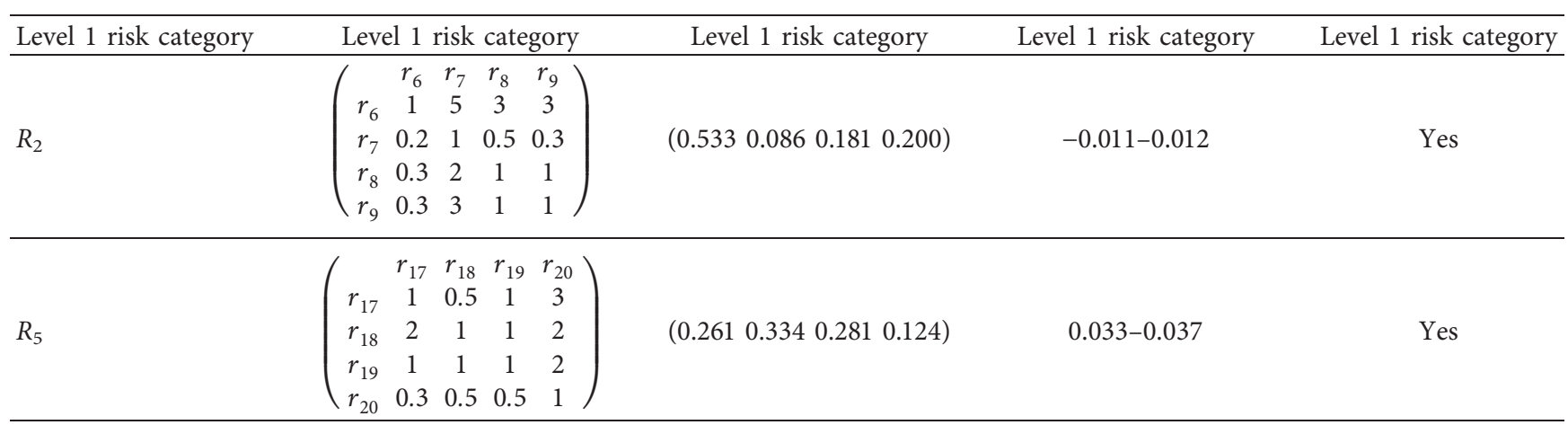

TABLE 5: Risk system coefficients of integrated pipeline gallery.

\begin{tabular}{|c|c|c|c|c|c|c|c|c|c|c|c|c|c|c|c|c|c|c|c|c|}
\hline & $r_{1}$ & $r_{2}$ & $r_{3}$ & $r_{4}$ & $r_{5}$ & $r_{6}$ & $r_{7}$ & $r_{8}$ & $r_{9}$ & $r_{10}$ & $r_{11}$ & $r_{12}$ & $r_{13}$ & $r_{14}$ & $r_{15}$ & $r_{16}$ & $r_{17}$ & $r_{18}$ & $r_{19}$ & $r_{20}$ \\
\hline$r_{1}$ & & & & & & & & & & & & & & & & & & & & \\
\hline$r_{2}$ & & & & & 0.38 & & & & & & & & & & & & & & & 0.222 \\
\hline$r_{3}$ & & & & & & & & & & & & & & & & & & & & \\
\hline$r_{4}$ & & & & & & & & & & & & & & & & & & & & \\
\hline$r_{5}$ & & & & & & & & & & & & & & & & & & & & \\
\hline$r_{6}$ & & & & & & & & & & & & & & & & & & & & \\
\hline$r_{7}$ & & & & & & & & & & & & & & & & & & & & \\
\hline$r_{8}$ & & & & & & & & & & & & & & & & & & & & \\
\hline$r_{9}$ & & & & 0.16 & & & & & & & & & & & & & & & & \\
\hline $\begin{array}{l}r_{10} \\
r_{1}\end{array}$ & & & 0.5 & & & & & & & 0.127 & & & & & 0.667 & & & & & \\
\hline$r_{12}$ & 0.5 & & & & 0.24 & & & & & & & & & & & & & & & 1 \\
\hline$r_{13}$ & & & & & & & & & & & & & & & & & & & 1 & \\
\hline$r_{14}$ & & & & & & & & & & & & & & & & & & & & \\
\hline$r_{15}$ & & & & 0.333 & & & & & & & & & & & & & & & & \\
\hline$r_{16}$ & & & & & & 1 & & & & 0.633 & 0.24 & & & & & & & & & \\
\hline$r_{17}$ & & & & & & & & & & & & & & & & & & & & \\
\hline$r_{18}$ & & & & & & & & & & & & & & & & & 1 & & & \\
\hline$r_{19}$ & & & & & & & & & & & & & & & & 1 & & 1 & & \\
\hline$r_{20}$ & & & & & & & & & & & & & & & & 1 & & & & \\
\hline
\end{tabular}

5.1. Building a Dynamic Model. System dynamics (abbreviated as SD) is a combination of the qualitative method and quantitative method, by constructing a physical environment close to reality, for the purpose of determining the relationship between various quantities and performing data simulation through a computer to obtain data that simulates the real scene to describe the system. System dynamics appeared in 1956, and its founder was Professor JW Forrester of Massachusetts Institute of Technology (MIT). System dynamics is the first system simulation method proposed by Professor Forrest in 1958 to analyze production management and inventory management and other enterprise problems. It was originally called industrial dynamics. The current application of system dynamics is no longer satisfied with the industrial field. Applications include learning organization, logistics, and supply chain management. The field of corporate management strategy and many other aspects is a discipline that analyzes and studies information feedback systems and is also a cross-integrated discipline that recognizes and solves system problems.

To establish the system dynamics model, we must first determine the causal circuit diagram of each factor. This article specifically constructs the circuit diagram for the comprehensive risk $R$ of the pipe gallery, the first-level risk $R_{i}$, and the second-level risk $r_{i}$. The construction process is divided into three steps:

(1) Determine the causal loop between comprehensive risk and first-level risk. Specifically, it links all primary risks with comprehensive risks.

(2) Determine the causal loop between the first-level risk and the second-level risk. Specifically, it is to connect the secondary risks under all primary risks with the corresponding primary risks.

(3) Determine the causal circuit between secondary risks. Specifically, it is connected according to the risk potential relationship path obtained through the analysis in the previous chapter.

After the above three steps, the risks are connected one by one to obtain the causal loop diagram of the comprehensive risk of the pipe gallery. This article uses AnyLogic software to establish the simulation model, and the final causal loop diagram is shown in Figure 1. 


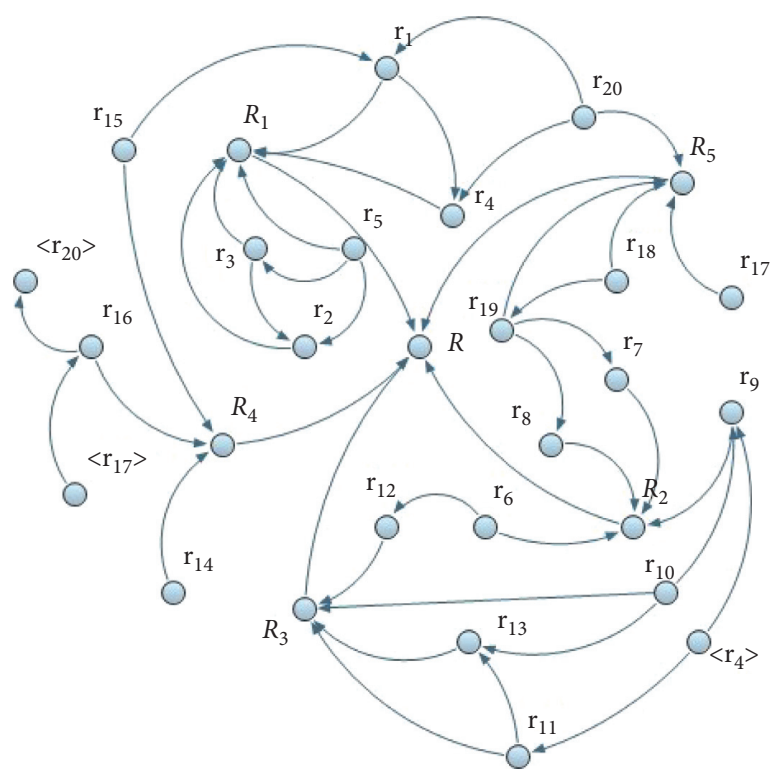

Figure 1: Causal circuit diagram.

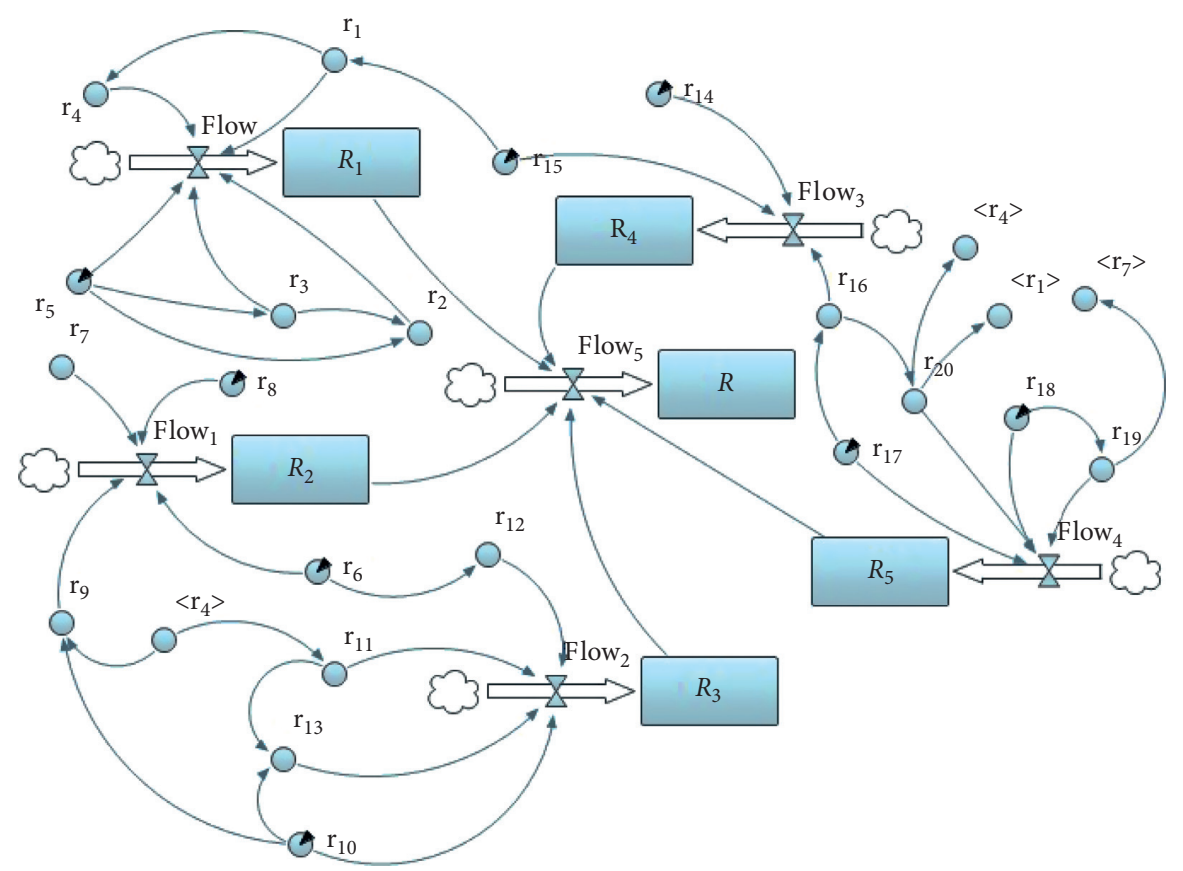

FIGURE 2: Stock flowchart.

Through the causal circuit diagram obtained, the logical management of each risk can be clearly sorted out. However, for the simulation of the overall risk of the integrated pipeline gallery and quantitative analysis, it is not enough to get the causal circuit diagram. It is also necessary to pass the cause and effect circuit diagram. The correlation relationship of each element in the loop diagram is combined with the weight vector corresponding to each risk to draw the stock flow diagram and construct the physical environment in the relevant simulation software. In this way, more accurate results can be obtained by quantifying the relationship between various risks.
The method steps for constructing the stock flow diagram are basically the same as the previous method's steps for constructing the causal logic diagram, except that when constructing the stock flow diagram, the weight matrix of each risk obtained through the risk structure matrix analysis in the previous chapter needs to be given. The path between risks is assigned. Figure 2 shows the final risk stock flowchart of the integrated pipeline gallery.

5.2. Analysis of Simulation Results. According to the risk simulation model diagram of the comprehensive pipeline 
corridor constructed in the previous part (data are seen in Table 6), it can be seen that, on the limited range of the model, the entire system has a total of eight parameters consisting of $r_{5}, r_{6}, r_{8}, r_{10}, r_{14}, r_{15}, r_{17}$, and $r_{18}$, and the remaining risks exist in the system as dynamic variables. It can be understood that the remaining risk factors are based on the degree of these eight risks and through various paths in the model to carry out weighting iterations, thus forming the entire complete system.

Sensitivity analysis is a method to study and analyze the sensitivity of the state or output changes of a system (or model) to changes in system parameters or surrounding conditions. Generally, sensitivity analysis is used in the optimization method to study the change of the basic data to the entire research result, so as to understand the degree of influence of the fluctuation of the original data on the entire research result. In the field of simulation modeling, sensitivity analysis can also determine which parameters have a greater impact on the system or model. Therefore, sensitivity analysis can analyze the degree of influence of the basic variables in the system on the entire system.

Therefore, according to the comprehensive pipeline gallery risk system constructed in this paper, sensitivity analysis and testing can be performed on the eight parameters that constitute the system, so as to determine the degree of influence of these basic risks on the entire pipeline gallery risk. This article still uses AnyLogic software. After constructing a complete system stock flow diagram, the sensitivity analysis of several parameters is directly performed on the basis of the complete model by changing the variable parameters in equal steps. Assuming that the basic parameter of each parameter is 1, the step size for sensitivity analysis is 1, and each step is increased. By sorting out the results of the sensitivity analysis of several variables, we have obtained the line graph of the sensitivity to the overall integrated risk $\boldsymbol{R}$ of the pipe gallery when the eight variable steps are increased twice in sequence, as shown in Figure 3 (the series represents the simulated experiment mark, the ordinate indicates the risk result, and the abscissa indicates the risk factor, the same below).

According to the results of the sensitivity analysis, after two step changes, $r_{15}$ has the largest comprehensive risk change rate for the pipe gallery, reaching $66.1 \%$, and the changes between $r_{10}$ and $r_{14}$ are the least significant, with the change rate being $0.05 \%$, and the rest rates of the change of several risks are $\mathrm{r} 5-26.5 \%$; r6-41.7\%; r8-18.3\%; r17-57.4\%; r18-40.5\%.

Because this article analyzes the risks in the operation and maintenance of the integrated pipeline gallery by building a model to simulate and intends to explore the risk factors that have the greatest impact on the overall risk of the integrated pipeline gallery, or the entire line, it is now the most sensitive. Risk $r_{15}$ above-ground and underground information is not linked as the main research object, and the impact of the second-level risk $r_{15}$ on the five first-level risks $R 1-R_{5}$ is analyzed. The final result of the simulation is shown in Figure 4.

From the content of the figure, it can be seen that the change of $r_{15}$ above-ground and underground information
TABLE 6: The risk simulation model data of the comprehensive pipeline corridor.

\begin{tabular}{|c|c|c|}
\hline $\mathrm{NCV}_{i} / \mathrm{BCV}_{i}$ & Judgment matrix & Weight vector \\
\hline $\mathrm{NCV}_{1}$ & $\left(\begin{array}{ccc} & r_{15} & r_{20} \\
r_{15} & 1 & 2 \\
r_{20} & 0.5 & 1\end{array}\right)$ & 0.6670 .333 \\
\hline $\mathrm{NCV}_{2}$ & $\left(\begin{array}{lll}r_{3} & 1 & 1 \\
r_{5} & 1 & 1\end{array}\right)$ & 0.50 .5 \\
\hline $\mathrm{NCV}_{3}$ & $r_{5}$ & 1 \\
\hline $\mathrm{NCV}_{4}$ & $\left(\begin{array}{ccc} & r_{1} & r_{20} \\
r_{1} & 1 & 1 \\
r_{20} & 1 & 1\end{array}\right)$ & 0.50 .5 \\
\hline $\mathrm{NCV}_{5}$ & - & - \\
\hline $\mathrm{NCV}_{6}$ & - & - \\
\hline $\mathrm{NCV}_{7}$ & $R_{19}$ & 1 \\
\hline $\mathrm{NCV}_{8}$ & - & - \\
\hline $\mathrm{NCV}_{9}$ & $\left(\begin{array}{ccc} & r_{4} & r_{10} \\
r_{4} & 1 & 0.3 \\
r_{10} & 3 & 1\end{array}\right)$ & 0.240 .76 \\
\hline $\mathrm{NCV}_{10}$ & - & - \\
\hline $\mathrm{NCV}_{11}$ & $R_{4}$ & 1 \\
\hline $\mathrm{NCV}_{12}$ & $R_{6}$ & 1 \\
\hline $\mathrm{NCV}_{13}$ & $\left(\begin{array}{ccc} & r_{10} & r_{11} \\
r_{10} & 1 & 3 \\
r_{11} & 0.3 & 1\end{array}\right)$ & 0.760 .24 \\
\hline $\mathrm{NCV}_{14}$ & - & - \\
\hline $\mathrm{NCV}_{15}$ & - & - \\
\hline $\mathrm{NCV}_{16}$ & $R_{17}$ & 1 \\
\hline $\mathrm{NCV}_{17}$ & - & - \\
\hline $\mathrm{NCV}_{18}$ & - & - \\
\hline $\mathrm{NCV}_{19}$ & $R_{18}$ & 1 \\
\hline $\mathrm{NCV}_{20}$ & $R_{16}$ & 1 \\
\hline $\mathrm{BCV}_{1}$ & $R_{4}$ & 1 \\
\hline $\mathrm{BCV}_{2}$ & - & - \\
\hline $\mathrm{BCV}_{3}$ & $R_{2}$ & 1 \\
\hline $\mathrm{BCV}_{4}$ & $\left(\begin{array}{ccc} & r_{9} & r_{11} \\
r_{9} & 1 & 2 \\
r_{11} & 0.5 & 1\end{array}\right)$ & 0.6670 .333 \\
\hline $\mathrm{BCV}_{5}$ & $\begin{array}{ccc} & r_{2} & r_{3} \\
r_{2} & 1 & 3\end{array}$ & 0.760 .24 \\
\hline $\mathrm{BCV}_{6}$ & $R_{12}$ & 1 \\
\hline $\mathrm{BCV}_{7}$ & - & - \\
\hline $\mathrm{BCV}_{8}$ & - & - \\
\hline $\mathrm{BCV}_{9}$ & - & - \\
\hline $\mathrm{BCV}_{10}$ & $\left.\begin{array}{ccc} & r_{9} & r_{13} \\
r_{9} & 1 & 0.2 \\
& 5 & 1\end{array}\right)$ & 0.1670 .833 \\
\hline $\mathrm{BCV}_{11}$ & $R_{13}$ & 1 \\
\hline $\mathrm{BCV}_{12}$ & - & - \\
\hline $\mathrm{BCV}_{13}$ & - & - \\
\hline $\mathrm{BCV}_{14}$ & - & - \\
\hline $\mathrm{BCV}_{15}$ & $R_{1}$ & 1 \\
\hline $\mathrm{BCV}_{16}$ & $R_{20}$ & 1 \\
\hline $\mathrm{BCV}_{17}$ & $R_{16}$ & 1 \\
\hline $\mathrm{BCV}_{18}$ & $R_{19}$ & 1 \\
\hline $\mathrm{BCV}_{19}$ & $R_{7}$ & 1 \\
\hline $\mathrm{BCV}_{20}$ & $\left(\begin{array}{ccc} & r_{1} & r_{3} \\
1 & 1 & 2 \\
r_{3} & 0.5 & 1\end{array}\right)$ & 0.6670 .333 \\
\hline
\end{tabular}




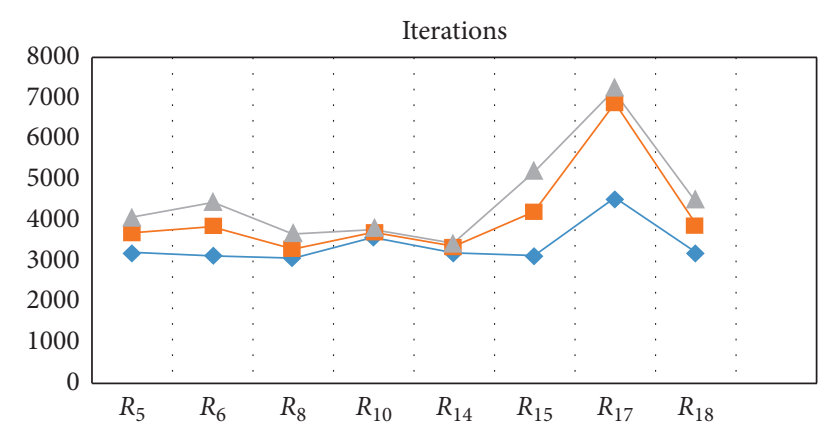

FIGURE 3: Sensitivity analysis of risk (1).

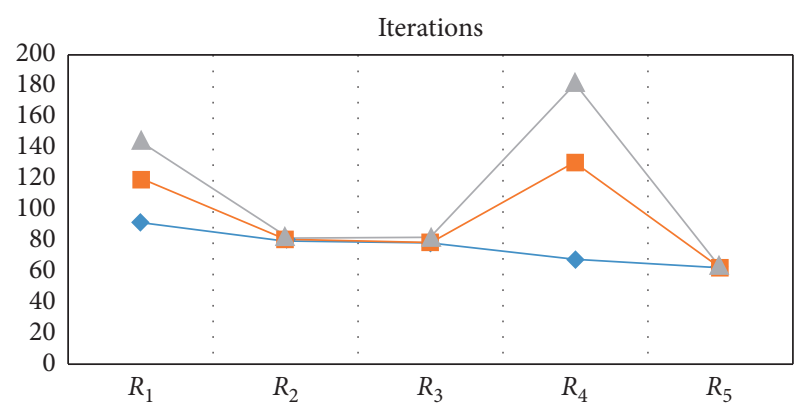

FIGURE 4: Sensitivity analysis of risk (2).

does not link the risk to the first-level risk $R_{1}$ management team risk and $R_{4}$ ancillary facility risk, but $r_{15}$ has a greater impact on $R_{2}$ environmental factor risk, $R_{3}$ gallery pipeline risk, and $R_{5}$; the influence of the risk of the pipe gallery itself is almost negligible.

According to the system's causal circuit diagram, it can be seen that the risk of unlinked information on the ground and underground has an impact on the risk of the management team through two risk paths, respectively: (1) $r_{15}$ above-ground and underground information nonlinkage risk $-r_{1}$ team information level risk $-R_{1}$ management team risk and (2) $r_{15}$ surface and underground information nonlinkage risk $-r_{1}$ team information level risk $-r_{4}$ information management level $-R_{1}$ management team risk and through the $r_{15}$ above-ground and underground information nonlinkage risk $-r_{20}$ management center and information transmission risk in the corridor $-R_{5}$ pipe corridor ontology risk has an impact on $R_{5}$.

However, through the causal loop diagram of the entire system, we can also find that there is a relationship path between $r_{15}$ and the risk of $R_{4}$ ancillary facilities, which can be understood as $r_{15}$ directly affects $R_{4}$. But, according to the sensitivity analysis of $r_{15}$ to $R_{4}$, the effect of $r_{15}$ to $R_{4}$ is not significant. Although according to quantitative analysis the path coefficient between $r_{15}$ and $R_{4}$ is 0.584 , the other two risk factors that directly affect $R_{4}$ are already at a relatively high level, but it may be due to several other factors that affect the level of $R_{4}$. The risk path has a more significant effect than $r_{15}$ through coupling, so the effect of $r_{15}$ on $R_{4}$ is not obvious.

Therefore, based on the research findings, the psychological state of people in the corridor environment, the impact of geological disasters, third-party construction damage, pipeline leakage, lighting, ventilation, drainage facilities, design, and construction that do not meet the operation and maintenance requirements, and uneven settlement caused by construction problems, supervisors need to attach great importance to the noninteraction of aboveground and underground information, especially the factors that do not interact with above-ground and underground information, the psychological state of personnel, the impact of geological disasters, third-party construction damage, design and construction not meeting the operation and maintenance requirements, and construction problems. As a result of uneven settlement, the frequency of supervision must be appropriately increased to prevent these major accident risks from causing serious damage. At the same time, combined with intelligent auxiliary facilities, when the supervision in the corridor exceeds the threshold, control measures can be taken as soon as possible to ensure the safe operation of the integrated pipeline corridor.

\section{Conclusion}

In Beijing and even across the country, such a background dominated by digital informatization has become an inevitable trend to realize the city's integrated pipeline corridor. Beijing actively responds to the country's call for the future construction plan of the pipe gallery. In accordance with the requirements of the country, Beijing has also ushered in a period of intensive construction of underground comprehensive pipe corridors. The Beijing City Master Plan (20162035) officially approved by the Central Committee of the Communist Party of China and the State Council is of great significance to the realization of the comprehensive, coordinated, and sustainable development of the capital city. The "comprehensive pipeline corridor" is an important basic guarantee for the safe operation of the city. One of the important means of sustainable urban development has been emphasized many times. By 2035, the underground comprehensive pipe gallery will reach about 450 kilometers. The backbone system of the underground comprehensive pipe gallery in the central urban area and the comprehensive pipe gallery system in key areas will be initially completed, and the scale effect of the underground comprehensive pipe gallery will further appear. In the future, how to conduct effective risk management after the comprehensive pipeline corridors in these cities are built and how to predict and respond to risks in advance will become a necessary research work.

This paper analyzes various typical risk factors of the integrated pipe gallery, synthesizes the previous research in this field, and applies a mathematical analysis combined with simulation modeling method to analyze the pipe gallery risk. The main research findings of this paper are as follows:

(1) Based on the literature analysis method, the frequency statistics of related articles in the two fields of integrated pipeline corridor operation and maintenance risk and project risk management are conducted, and the comprehensive pipeline corridor 
operation with 5 first-level risks and 20 second-level risks is preliminarily summarized. The risk list in the maintenance management can be used as a basis for the subsequent research on the risk of the integrated pipeline corridor operation and maintenance management, and it is convenient to organize the basic risk factors.

(2) This paper applies a method based on the combination of analytic hierarchy process and risk structure matrix method. Through mathematical analysis and deduction, a set of weights based on each risk in the previous risk list is obtained, and each risk is determined according to the risk matrix. The risk path between the risks is drawn, and the causal circuit diagram between the risks is obtained. While the potential relationship between the various risks can be clearly clarified, it is necessary to build the risk stock flow diagram in the simulation software. Simulation experiments are used as preparatory work.

(3) This article uses a combination of multiple methods to establish a system dynamics model for the risk of a comprehensive pipeline gallery and conducts simulation experiments on the model. Through multiple sensitivity analyses of parameters, the key risk factors are obtained. Several risk paths that seriously affect the overall risk level are proposed, providing relevant basis for dealing with risks in the daily operation and maintenance management of the integrated pipeline gallery in the future.

This study has positive significance for dealing with the security risk problem of urban underground integrated pipeline corridor pipeline with complexity and uncertainty. On the one hand, in view of the complex composition of risk elements related to integrated pipeline corridors and the complex relationship between elements, the main components of information security risk sources are analyzed by using rich textual information, combined with expert consultation, and the hierarchical structure relationship between risk elements is clarified, and a clear, structured, and systematic index system of integrated pipeline corridor safety risk assessment is constructed; on the other hand, in view of the uncertainty in the evolution of integrated pipeline corridor safety risk and derivation process, we cut from the perspective of probability and use the applicability of system dynamics network in dealing with uncertainty problems and apply it to the quantitative study of safety risk assessment of urban underground integrated pipeline corridors.

\section{Data Availability}

The test data used to support the findings of this study are available from the corresponding author upon request.

\section{Conflicts of Interest}

The authors declare that they have no conflicts of interest.

\section{References}

[1] C. Francesca and D. Matjaž, "A simplified risk-targeted decision model for the verification of the seismic performance of critical infrastructure components to the operational limit state," Engineering Structures, vol. 204, 2020.

[2] Y. J. Chen, H. Y. Li, W. J. Wang, B. Xue, and G. X. Li, "Disaster risk analysis of integrated pipeline corridor operation and maintenance based on Bayesian network," Journal of Safety and Environment, vol. 18, no. 6, pp. 2109-2114, 2018.

[3] Q. Li, W. Duan, and G. Q. Xu, "Research on risk factors of operation and maintenance management of comprehensive pipe gallery based on DEMATEL," Tunnel Construction (Chinese and English), vol. 39, no. 1, pp. 31-39, 2019.

[4] Y. Jang and S. Jung, "Quantitative risk assessment for gasexplosion at buried common utility tunnel," Journal of the Korean Institute of Gas, vol. 20, no. 5, pp. 89-95, 2016.

[5] Y. H. Wang, "Study on fire risk factors of operation and maintenance of urban underground comprehensive pipe gallery," Value Engineering, vol. 38, no. 5, pp. 57-60, 2019.

[6] S. Ghosh and J. Jintanapakanont, "Identifying and assessing the critical risk factors in an underground rail project in Thailand: a factor analysis approach," International Journal of Project Management, vol. 22, no. 8, pp. 633-643, 2004.

[7] M. Eybpoosh, I. Dikmen, and B. M. Talat, "Identification of risk paths in international construction projects using structural equation modeling," Journal of Construction Engineering and Management, vol. 137, no. 12, pp. 1164-1175, 2011.

[8] K. F. Chien, Z. H. Wu, and S. C. Huang, "Identifying and assessing critical risk factors for BIM projects: empirical study," Automation in Construction, vol. 45, pp. 1-15, 2014.

[9] J. L. Yang and G. H. Tzeng, "An integrated MCDM technique combined with DEMATEL for a novel cluster-weighted with ANP method," Expert Systems with Applications, vol. 38, no. 3, pp. 1417-1424, 2011.

[10] W. H. Tsai and W. C. Chou, "Selecting management systems for sustainable development in SMEs: a novel hybrid model based on DEMATEL, ANP, and ZOGP," Expert Systems with Applications, vol. 36, no. 2, pp. 1444-1458, 2009.

[11] W. W. Wu, "Choosing knowledge management strategies by using a combined ANP and DEMATEL approach," Expert Systems with Applications, vol. 35, no. 3, pp. 828-835, 2008.

[12] J. C. Perello and J. C. Esparza, "Assessing governance issues of urban utility tunnels," Tunnelling and Underground Space Technology incorporating Trenchless Technology Research, vol. 33, 2013.

[13] J. C. Perello and J. C. Esparza, "An analysis of utility tunnel viability in urban areas," Civil Engineering and Environmental Systems, vol. 23, no. 1, 2006.

[14] C. Julian, C. Jorge, and C. Vicente, "Criticality and threat analysis on utility tunnels for planning security policies of utilities in urban underground space," Expert Systems with Applications, vol. 40, no. 11, 2013.

[15] W. Wang and J. Fang, "Study on the risk evaluation model of utility tunnel project under a PPP mode," in Proceedings of International Conference on Construction and Real Estate Management, Guangzhou, China, November 2017.

[16] L. He, G. Ma, Q. Hu et al., "A novel method for risk assessment of cable fires in utility tunnel," Mathematical Problems in Engineering, vol. 2019, Article ID 2563012, 14 pages, 2019.

[17] X.-X Ding, K. Liu, S.-j Shi et al., "Risk assessment of PPP model based on fault tree model for urban underground 
utility tunnel," in Proceedings of the 2019 International Conference on Civil Engineering, Mechanics and Materials Science (CEMMS 2019), Changsha, China, August 2019.

[18] L. Rita and H. Herbert, "Risk analysis during tunnel construction using Bayesian networks: porto metro case study," Tunnelling and Underground Space Technology, vol. 27, no. 1, pp. 86-100, 2012.

[19] K. Golam, T. Solomon, and F. Alex, "Evaluating risk of water mains failure using a Bayesian belief network model," European Journal of Operational Research, vol. 240, no. 1, pp. 220-234, 2015.

[20] Z. Limao, X. Wu, Q. Yawei, M. J. Skibniewski, and W. Liu, "Towards a fuzzy Bayesian network based approach for safety risk analysis of tunnel-induced pipeline damage," Risk Analysis, vol. 36, no. 2, pp. 278-301, 2016.

[21] M. M. Khwaja, P. C. C. Albert, Z. Hafiz, I. K. Mohsen, and E. A. Ernest, "Fuzzy integral-based risk-assessment approach for public-private partnership infrastructure projects," Journal of Construction Engineering and Management, vol. 144, no. 12, Article ID 04018111, 2018.

[22] H. Wu, M. Wang, and J. Wang, "Shield construction safety risk assessment of metro tunnels based on cloud model," in Proceedings of International Conference on Urban Engineering and Management Science, April 2020.

[23] L. Kang, "Risk assessment of urban underground utility tunnel based on fuzzy analytic hierarchy process," Operations Research and Fuzziology, vol. 9, no. 2, pp. 131-139, 2019.

[24] W. K. Marian, G. Beata, K. Adam, and S. Agata, "Risk modelling with bayesian networks-case study: construction of tunnel under the dead vistula river in gdansk," Procedia Engineering, vol. 196, 2017.

[25] J. Martinka, P. Rantuch, J. Sulová, and F. Martinka, “Assessing the fire risk of electrical cables using a cone calorimeter," Journal of Thermal Analysis and Calorimetry, vol. 135, no. 6, pp. 3069-3083, 2019.

[26] S. Fan, E. B. Davis, Z. Yang, J. Zhang, and X. Yan, "Incorporation of human factors into maritime accident analysis using a data-driven Bayesian network," Reliability Engineering and System Safety, vol. 203, 2020. 